\title{
An alternative to Dirichlet-to-Neumann maps for waveguides
}

\author{
Anne-Sophie Bonnet-Ben Dhia Guillaume Legendre
}

May 31, 2011

\begin{abstract}
We are interested by the treatment of the radiation condition at infinity for the numerical solution of a problem set in an unbounded waveguide. We propose an alternative to the classical approach involving a modal expression of Dirichlet-to-Neumann (DtN) operators. This method is particularly simple to implement since it only requires the solution of boundary value problems with local boundary conditions. The corresponding approximate solution is comparable in accuracy to the one obtained by truncating the infinite series in the DtN maps.
\end{abstract}

\section{The diffraction problem in a semi-infinite waveguide}

In this note, we consider, for the sake of simplicity, an acoustic scattering problem set in a possibly locally perturbed semi-infinite waveguide. More precisely, we consider a connected unbounded domain $\Omega \subset \mathbb{R}^{d}$ with $d \geq 2$, such that $\Omega \cap\left\{\boldsymbol{x}=\left(\boldsymbol{x}_{S}, x_{d}\right) \mid x_{d}>0\right\}=\left\{\boldsymbol{x}=\left(\boldsymbol{x}_{S}, x_{d}\right) \mid \boldsymbol{x}_{S} \in S, x_{d}>0\right\}$, where $S$ is a bounded subset of $\mathbb{R}^{d-1}$, and $\Omega \cap\left\{\boldsymbol{x}=\left(\boldsymbol{x}_{S}, x_{d}\right) \mid x_{d}<0\right\}=\Omega_{0}$ is a bounded domain (see Figure 1). We are interested in numerically solving the following boundary value problem

$$
-\Delta u-k^{2} u=f \text { in } \Omega, \partial_{\boldsymbol{n}} u=0 \text { on } \partial \Omega,
$$

supplemented by a radiation condition at infinity. It is assumed that the source term $f$ belongs to $L^{2}(\Omega)$ and is compactly supported in $\Omega_{0}$, and that the wave number $k$ is real. The vector $\boldsymbol{n}$ denotes the outward unit normal on $\partial \Omega$.

The prescribed radiation condition states that the solution is "outgoing" at infinity. It can be written down by introducing the so-called modes of the guide, which are functions with separated variables of the form $\varphi_{n}\left(\boldsymbol{x}_{s}\right) e^{ \pm \mathrm{i} \beta_{n} x_{d}}, n \in \mathbb{N}$, the complex numbers $\beta_{n}$ being such that $\beta_{n}=$ $\sqrt{k^{2}-\lambda_{n}}$ for $k^{2} \geq \lambda_{n}$ and $\beta_{n}=i \sqrt{\lambda_{n}-k^{2}}$ for $k^{2} \leq \lambda_{n}$. Here, the real positive scalar $\lambda_{n}$ denotes the $n^{\text {th }}$ eigenvalue of the negative Laplace operator acting in $L^{2}(S)$ and associated with the homogeneous Neumann boundary condition on $\partial S$, and the orthonormal real-valued functions $\varphi_{n}$ are the corresponding eigenfunctions. A mode is said to be propagative if $\beta_{n} \in \mathbb{R}$, and evanescent if $\beta_{n} \in i \mathbb{R}$ (note that there is a finite number $N_{\text {prop }}$ of propagative modes). If $k^{2} \neq \lambda_{n}, \forall n \in \mathbb{N}$,



Figure 1: A realization of the domain $\Omega$. 
saying the solution is outgoing simply means that, for any $\boldsymbol{x}$ in $\Omega$ such that $x_{d}>\ell \geq 0$, the field $u(\boldsymbol{x})$ is given by a convergent series of rightgoing modes $\varphi_{n}\left(\boldsymbol{x}_{S}\right) e^{\mathrm{i} \beta_{n} x_{d}}, n \in \mathbb{N}$, that is

$$
u(\boldsymbol{x})=\sum_{n=0}^{+\infty} A_{n}^{+}(\ell, u) \varphi_{n}\left(\boldsymbol{x}_{S}\right) e^{\mathrm{i} \beta_{n}\left(x_{d}-\ell\right)}, \forall \boldsymbol{x}_{S} \in S, \forall x_{d}>\ell \geq 0 .
$$

In other words, the amplitudes $A_{n}^{-}(\ell, u), n \in \mathbb{N}$, of the solution $u$ on the leftgoing modes $\varphi_{n}\left(\boldsymbol{x}_{S}\right) e^{-\mathrm{i} \beta_{n}\left(x_{d}-\ell\right)}$ must vanish. Notice that it follows from the orthonormality of the functions $\varphi_{n}, n \in \mathbb{N}$, that $A_{n}^{+}(\ell, u)=\left(u(\cdot, \ell), \varphi_{n}\right)_{S}$, where $u\left(\cdot, x_{d}\right)$ denotes the function $u$ viewed as a function of the variable $\boldsymbol{x}_{S}$ and $(\cdot, \cdot)_{S}$ denotes the scalar product on $L^{2}(S)$.

For both theoretical and numerical purposes, it is convenient to replace problem (1) by an equivalent problem set on a bounded domain $\Omega_{\ell}=\Omega \cap\left\{\boldsymbol{x}=\left(\boldsymbol{x}_{S}, x_{d}\right) \mid \boldsymbol{x}_{S} \in S, x_{d}<\ell\right\}$, with $\ell \geq 0$. This is achieved by incorporating, using a Dirichlet-to-Neumann (DtN) map, the radiation condition into an exact nonlocal boundary condition on the artificial boundary $S_{\ell}=S \times\{\ell\}$, that is

$$
\partial_{\boldsymbol{n}} u=T_{\ell}(u)=\mathrm{i} \sum_{n=0}^{+\infty} \beta_{n}\left(u(\cdot, \ell), \varphi_{n}\right)_{S} \varphi_{n} \text { on } S_{\ell} .
$$

Classical arguments allow to prove that the boundary value problem

$$
-\Delta u-k^{2} u=f \text { in } \Omega_{\ell}, \partial_{\boldsymbol{n}} u=0 \text { on } \partial \Omega \cap \partial \Omega_{\ell}, \partial_{\boldsymbol{n}} u-T_{\ell}(u)=0 \text { on } S_{\ell},
$$

is of Fredholm type. In what follows, we assume that uniqueness holds so that the problem is well-posed for every $\ell \geq 0$. Now, to compute an approximate solution to problem (3), one can discretize its variational formulation by a finite element method and truncate the infinite series in $T_{\ell}$ (see $[1,2]$ ). It can be seen that the rank of truncation, which is always greater or equal to $N_{\text {prop }}$, can be chosen smaller by increasing the length $\ell$. A drawback of this approach is the nonlocality of the boundary condition, which makes both the implementation of this method more difficult and the numerical solution of the associated algebraic system more expensive, the resulting matrix not being fully sparse. Here, we propose an alternative approach, which relies solely on the solution of problems with local boundary conditions, and thus workable into any finite element code.

\section{The effect of replacing the transparent condition by a Robin condition}

Let us consider the following boundary value problem

$$
-\Delta u_{r}-k^{2} u_{r}=f \text { in } \Omega_{\ell}, \partial_{\boldsymbol{n}} u_{r}=0 \text { on } \partial \Omega \cap \partial \Omega_{\ell}, \partial_{\boldsymbol{n}} u_{r}-\mathrm{i} \alpha u_{r}=0 \text { on } S_{\ell},
$$

where $\alpha \in \mathbb{R} \backslash\{0\}$ is a given parameter. Note that such a problem is well-posed; indeed, it satisfies the Fredholm alternative and the uniqueness is a consequence of Holmgren's theorem.

To compare $u_{r}$ with the solution $u$ to (1), we proceed as in [3] and derive an equivalent problem satisfied by $u_{r}$ in the subdomain $\Omega_{0}$ (we emphasize that this problem, which involves a nonlocal boundary condition, is a theoretical tool never to be used numerically). Knowing that the solution to (4) can be expanded on the guided modes as follows

$$
u_{r}(\boldsymbol{x})=\sum_{n=0}^{+\infty}\left(A_{n}^{+}\left(0, u_{r}\right) e^{\mathrm{i} \beta_{n} x_{d}}+A_{n}^{-}\left(0, u_{r}\right) e^{-\mathrm{i} \beta_{n} x_{d}}\right) \varphi_{n}\left(\boldsymbol{x}_{S}\right), \quad \forall \boldsymbol{x} \in S \times[0, \ell],
$$

and using the boundary condition on $S_{\ell}$, one has

$$
\mathrm{i} \beta_{n}\left(A_{n}^{+}\left(0, u_{r}\right) e^{\mathrm{i} \beta_{n} \ell}-A_{n}^{-}\left(0, u_{r}\right) e^{-\mathrm{i} \beta_{n} \ell}\right)=\mathrm{i} \alpha\left(A_{n}^{+}\left(0, u_{r}\right) e^{\mathrm{i} \beta_{n} \ell}+A_{n}^{-}\left(0, u_{r}\right) e^{-\mathrm{i} \beta_{n} \ell}\right), \forall n \in \mathbb{N},
$$

and

$$
\frac{A_{n}^{-}\left(0, u_{r}\right)}{A_{n}^{+}\left(0, u_{r}\right)}=\frac{\beta_{n}-\alpha}{\beta_{n}+\alpha} e^{2 \mathrm{i} \beta_{n} \ell}=R_{n}(\ell), \forall n \in \mathbb{N}
$$


from which we obtain that $u_{r}$ satisfies the condition:

$$
\partial_{\boldsymbol{n}} u_{r}-\mathrm{i} \sum_{n=0}^{+\infty} \beta_{n} \frac{1-R_{n}(\ell)}{1+R_{n}(\ell)}\left(u_{r}(\cdot, 0), \varphi_{n}\right)_{S} \varphi_{n}=0 \text { on } S_{0} .
$$

The coefficient $R_{n}(\ell)$ gives a measure of the reflection on the $n^{\text {th }}$ mode caused by the truncation of the domain and the use of a Robin boundary condition at $x_{d}=\ell$. It vanishes if $\alpha=\beta_{n}$ and is otherwise nonzero, but it can be made arbitrarily small by choosing $\ell$ sufficiently large when the $n^{\text {th }}$ mode is evanescent. Owing to this remark, the problem satisfied by $u_{r}$ in $\Omega_{0}$ can be rewritten as follows

$$
-\Delta u_{r}-k^{2} u_{r}=f \text { in } \Omega_{0}, \partial_{\boldsymbol{n}} u_{r}=0 \text { on } \partial \Omega \cap \partial \Omega_{0}, \partial_{\boldsymbol{n}} u_{r}-T_{0}\left(u_{r}\right)=L_{\ell}^{N}\left(u_{r}\right)+S_{\ell}^{N}\left(u_{r}\right) \text { on } S_{0} .
$$

In the above problem, the difference between the "exact" boundary condition, which involves the DtN operator $T_{0}$ (consider (3) with $\ell=0$ ), and the approximate one has been split into two contributions respectively defined by

$$
L_{\ell}^{N}(u)=\mathrm{i} \sum_{n=0}^{N-1} \frac{2 \beta_{n} R_{n}(\ell)}{1+R_{n}(\ell)}\left(u(\cdot, 0), \varphi_{n}\right)_{S} \varphi_{n} \text { and } S_{\ell}^{N}(u)=\mathrm{i} \sum_{n=N}^{+\infty} \frac{2 \beta_{n} R_{n}(\ell)}{1+R_{n}(\ell)}\left(u(\cdot, 0), \varphi_{n}\right)_{S} \varphi_{n},
$$

where $N$ is an integer such that

$$
\left\|S_{\ell}^{N}\right\|_{\mathcal{L}\left(H^{1 / 2}(S), H^{-1 / 2}(S)\right)} \leq C \max _{n \geq N}\left|\frac{R_{n}(\ell)}{1+R_{n}(\ell)}\right| \sim C e^{-2 \operatorname{Im}\left(\beta_{N} \ell\right)} \leq \varepsilon,
$$

with $\varepsilon>0$ a prescribed tolerance. Observe that we must have $N \geq N_{\text {prop }}$ for the last inequality in (8) to be satisfied, as we use the fact that $\left|R_{n}(\ell)\right|$ decreases exponentially as $\left|\beta_{n} \ell\right|$ increases if the $n^{\text {th }}$ mode is evanescent.

\section{The auxiliary fields and the recomposed approximate so- lution}

Since the operator $S_{\ell}^{N}$ can be rendered negligible, the discrepancy between the approximate solution $u_{r}$ and the actual solution $u$ is mainly due to the operator $L_{\ell}^{N}$. Our idea is to use both the fact that $L_{\ell}^{N}$ is of finite rank $N$ and the linearity of the problem to construct a new approximate solution $\widetilde{u}$ verifying

$$
-\Delta \widetilde{u}-k^{2} \widetilde{u}=f \text { in } \Omega_{0}, \partial_{\boldsymbol{n}} \widetilde{u}=0 \text { on } \partial \Omega \cap \partial \Omega_{0}, \partial_{\boldsymbol{n}} \widetilde{u}-T_{0}(\widetilde{u})=S_{\ell}^{N}(\widetilde{u}) \text { on } S_{0} .
$$

One can show, taking inspiration from [3], that, for $\varepsilon$ small enough (that is, for $\ell$ and/or $N$ large enough), problem (9) is well-posed and that we have the estimate $\|u-\widetilde{u}\|_{H^{1}\left(\Omega_{0}\right)} \leq C \varepsilon$, where $C$ is a constant independent of both $\ell$ and $N$.

To effectively build the approximation $\widetilde{u}$, we set $\widetilde{u}=u_{r}+u_{c}$, the corrective field $u_{c}$ being, by linearity, solution to

$$
-\Delta u_{c}-k^{2} u_{c}=0 \text { in } \Omega_{0}, \partial_{\boldsymbol{n}} u_{c}=0 \text { on } \partial \Omega \cap \partial \Omega_{0}, \partial_{\boldsymbol{n}} u_{c}-T_{0}\left(u_{c}\right)-S_{\ell}^{N}\left(u_{c}\right)=-L_{\ell}^{N}\left(u_{r}\right) \text { on } S_{0} .
$$

Since the range of $L_{\ell}^{N}$ is included in the $N$-dimensional vector space $V_{N}=\operatorname{span}\left\{\varphi_{0}, \ldots, \varphi_{N-1}\right\}$, we prove that this correction may be written down as a linear combination of $N$ functions $u^{(j)}$, $j=0, \ldots, N-1$, satisfying $-\Delta u^{(j)}-k^{2} u^{(j)}=0$ in $\Omega_{0}, \partial_{\boldsymbol{n}} u^{(j)}=0$ on $\partial \Omega \cap \partial \Omega_{0}$, and such that the family $\left\{g^{(j)}\right\}_{j=0, \ldots, N-1}$ of functions defined by

$$
g^{(j)}=\left.\partial_{\boldsymbol{n}} u^{(j)}\right|_{S_{0}}-T_{0}\left(u^{(j)}\right)-S_{\ell}^{N}\left(u^{(j)}\right), j=0, \ldots, N-1 .
$$


form a basis of $V_{N}$. Assuming for a moment the existence of such functions $u^{(j)}$ (a particular choice is proposed below), we then have

$$
\widetilde{u}=u_{r}+\sum_{j=0}^{N-1} \mu^{(j)} u^{(j)},
$$

where the coefficients $\mu^{(j)}, j=0, \ldots, N-1$, are such that

$$
\sum_{j=0}^{N-1} \mu^{(j)} g^{(j)}=-L_{\ell}^{N}\left(u_{r}\right) .
$$

In practice, the fields $u^{(j)}, j=0, \ldots, N-1$, can be conveniently obtained as the solutions to

$$
-\Delta u^{(j)}-k^{2} u^{(j)}=0 \text { in } \Omega_{\ell}, \partial_{\boldsymbol{n}} u^{(j)}=0 \text { on } \partial \Omega \cap \partial \Omega_{\ell}, \partial_{\boldsymbol{n}} u^{(j)}-\mathrm{i} \alpha u^{(j)}=\varphi_{j} \text { on } S_{\ell},
$$

since this choice leads to a family of linearly independent functions $g^{(j)}, j=0, \ldots, N-1$, defined by (10). Indeed, suppose that $\sum_{j=0}^{N-1} \mu^{(j)} g^{(j)}=0$. Setting $u^{*}=\sum_{j=0}^{N-1} \mu^{(j)} u^{(j)}$ and using (10), we see that $u^{*}$ satisfies problem (9) with $f=0$. This problem being well-posed, one has $u^{*} \equiv 0$. In view of the definition (13) of the auxiliary fields $u^{(j)}, j=1, \ldots, N-1$, it follows that $0=$ $\partial_{\boldsymbol{n}} u^{*}-\mathrm{i} \alpha u^{*}=\sum_{j=0}^{N-1} \mu^{(j)} \varphi_{j}$ on $S_{\ell}$, which implies that $\mu^{(j)}=0, j=1, \ldots, N-1$.

\section{Implementation}

Summing up, the solution method we propose consists of computing successively, using finite element approximations (other discretization techniques are, of course, possible), the field $u_{r}$, which is solution to problem (4), the auxiliary fields $u^{(j)}, j=0, \ldots, N-1$, which solve the family of problems (13), and the coefficients $\mu^{(j)}, j=0, \ldots, N-1$, the approximate solution $\widetilde{u}$ being finally recomposed according to (11). We stress that these various computations do not require to build and solve $N+1$ algebraic systems associated with the finite element discretization, since they only differ by their respective right-hand sides. Hence, once the (sparse) matrix of the systems has been factorized, the solutions (which can moreover be performed in parallel) merely amount to forward and backward substitutions.

As it is impractical to compute the scalars $\mu^{(j)}, j=0, \ldots, N-1$ from system (12), we need to derive another linear system satisfied by these coefficients. To do so, notice that the second boundary condition in (9) imposes that the field $\partial_{\boldsymbol{n}} \widetilde{u}_{S_{S_{0}}}-T_{0}(\widetilde{u})$ is orthogonal to $V_{N}$, which means that no reflection occurs on the $N$ first guided modes. In particular, we have $A_{j}^{-}(0, \widetilde{u})=0$, $j=0, \ldots, N-1$, or, equivalently, $A_{j}^{-}(\ell, \widetilde{u})=A_{j}^{-}(0, \widetilde{u}) e^{-\mathrm{i} \beta_{m} \ell}=0, j=0, \ldots, N-1$. Therefore, using (11), we find that the scalars $\mu^{(j)}$ are the unique solution to the following linear system

$$
\sum_{i=0}^{N-1} \mu^{(i)} A_{j}^{-}\left(\ell, u^{(i)}\right)=-A_{j}^{-}\left(\ell, u_{r}\right), j=0, \ldots, N-1 .
$$

An advantage of the above system is that the computation of its $N(N+1)$ coefficients $A_{j}^{-}\left(\ell, u_{r}\right)$ and $A_{j}^{-}\left(\ell, u^{(i)}\right), i, j=0, \ldots, N-1$, is inexpensive. Indeed, using (and differentiating) (5) and the boundary condition satisfied by $u_{r}$ on $S_{\ell}$, it follows that

$A_{j}^{ \pm}\left(\ell, u_{r}\right)=\frac{1}{2}\left(\left(u_{r}(\cdot, \ell), \varphi_{j}\right)_{S} \pm \frac{1}{\mathrm{i} \beta_{j}}\left(\partial_{x_{d}} u_{r}(\cdot, \ell), \varphi_{j}\right)_{S}\right)=\frac{1}{2}\left(1 \pm \frac{\alpha}{\beta_{j}}\right)\left(u_{r}(\cdot, \ell), \varphi_{j}\right)_{S}, j=0, \ldots, N-1$.

A similar approach may be used to evaluate the coefficients $A_{j}^{ \pm}\left(\ell, u^{(i)}\right), i, j=0, \ldots, N-1$.

Finally, one obviously sees that taking $\alpha=\beta_{m}$ for a given index $m$ in $\{0, \ldots, N-1\}$ reduces by one unit the rank of the operator $L_{\ell}^{N}$, and therefore only $N-1$ auxiliary functions and $N-1$ associated coefficients need to be computed in that case. A natural choice is then $\alpha=\beta_{0}=k$. 


\section{Additional comments and possible extensions}

The present method is applicable to the case of an infinite guide, at the expense of introducing twice as many auxiliary fields and associated coefficients. It can also be used to solve diffraction problems by bounded scatterers in $\mathbb{R}^{d}$ by substituting spherical harmonics for modes. It is not restricted to acoustic or scalar waves; gravity, electromagnetic or elastic waves could have also been considered. However, in the later cases, the difficulty is that the transverse modes are generally not orthogonal. One may circumvent this problem by resorting to a biorthogonality framework (see [4] in an elastic waveguide setting for instance).

The Robin boundary condition (used in problems (4) and (13)) can be replaced by any other convenient homogeneous local boundary condition, as long as it leads to a well-posed problem for the field $u_{r}$. However, if a Dirichlet boundary condition is employed, the computation of the traces of normal derivatives on $S_{\ell}$, which are needed to obtain the coefficients of the algebraic system (14), requires some post-processing based on Green's formula.

Finally, the number of auxiliary fields to be computed, which has to be greater than the number of propagative guided modes, can be significantly reduced by combining the proposed approach with the perfectly matched layer (PML) technique. Actually, this solution method was initially devised to overcome the disastrous effects that the so-called backward waves have on the PMLs (see [5] for an example). These aspects, as well as numerical results, will be presented in a future publication.

\section{References}

[1] C. I. Goldstein, A finite element method for solving Helmholtz type equations in waveguides and other unbounded domains, Math. Comp. 39 (160) (1982) 309-324.

[2] I. Harari, I. Patlashenko, D. Givoli, Dirichlet-to-Neumann maps for unbounded wave guides, J. Comput. Phys. 143 (1) (1998) 200-223.

[3] E. Bécache, A.-S. Bonnet-Ben Dhia, G. Legendre, Perfectly matched layers for the convected Helmholtz equation, SIAM J. Numer. Anal. 42 (1) (2004) 409-433.

[4] V. Baronian, A.-S. Bonnet-Ben Dhia, E. Lunéville, Transparent boundary conditions for the harmonic diffraction problem in an elastic waveguide, J. Comput. Appl. Math. 234 (6) (2010) $1945-1952$.

[5] E. A. Skelton, S. D. Adams, R. V. Craster, Guided elastic waves and perfectly matched layers, Wave Motion 44 (7-8) (2007) 573-592. 